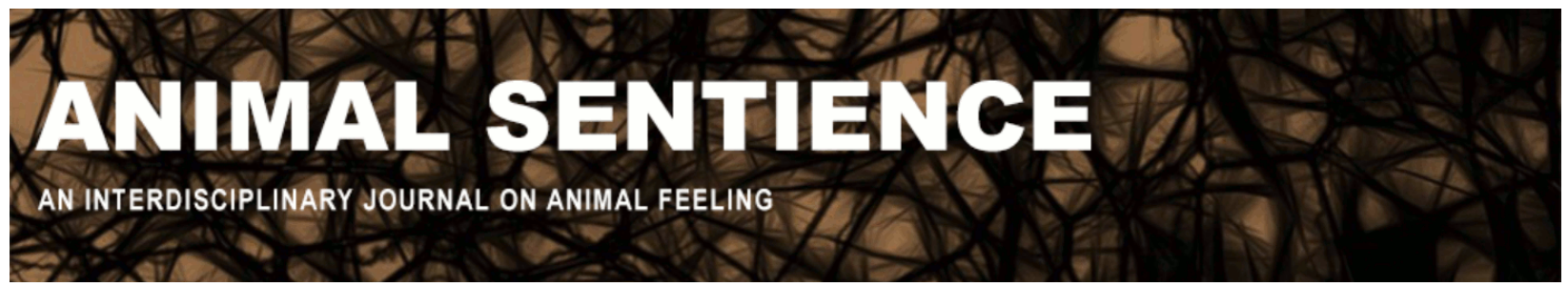

Morsella, Ezequiel and Walker, Erica B. (2016) What makes us conscious is not what makes us human. Animal Sentience 9(11)

DOI: $10.51291 / 2377-7478.1142$

Date of submission: 2016-08-12

Date of acceptance: 2016-08-18

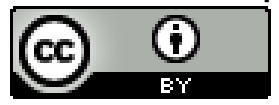

This article has appeared in the journal Animal

Sentience, a peer-reviewed journal on animal

cognition and feeling. It has been made open access,

free for all, by WellBeing International and deposited

in the WBI Studies Repository. For more information,

please contact

wbisr-info@wellbeingintl.org.

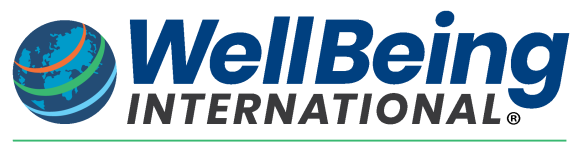

SOLUTIONS FOR PEOPLE, ANIMALS AND ENVIRONMENT 


\title{
What makes us conscious is not what makes us human
}

Commentary on Klein \& Barron on Insect Experience

\author{
Ezequiel Morsella \\ Department of Psychology, San Francisco State University \\ Department of Neurology, University of California, San Francisco \\ Erica B. Walker \\ Department of Psychology, San Francisco State University
}

\begin{abstract}
Consistent with the promising proposal of Klein \& Barron (K \& B), we discuss how what makes us conscious appears to be distinct from and more widespread in the animal kingdom than what distinguishes us from other species. Many of the abilities that do distinguish humans from other species (e.g., syntax and co-articulation in speech production) can be mediated unconsciously. The kind of functional architecture proposed by $K \& B$ may engender an "action selection bottleneck" in both humans and nonhuman species. As noted by K \& B, this bottleneck is intimately related to conscious processing.
\end{abstract}

Keywords: animal consciousness, action selection, passive frame theory

\begin{abstract}
Ezequiel Morsella, Director of the Action and Consciousness Laboratory, San Francisco State University (SFSU), does research on the differences between the conscious and unconscious brain circuits underlying human action production. http://online.sfsu.edu/morsella/people.html
\end{abstract}

Erica B. Walker, graduate student in the Department of Psychology, San Francisco State University, does research on the role of evolutionary forces in shaping human action control, working memory, and consciousness.
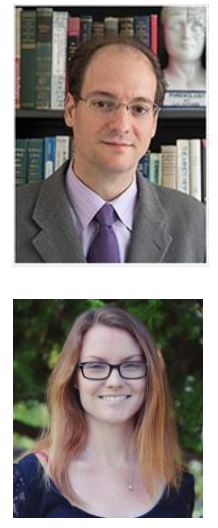

Which neural circuits give rise to conscious states? To address this question, Klein \& Barron (2016) (henceforth K \& B) use the clever strategy of examining the most basic instantiations of these states, including in nonhuman species. Their innovative approach accords well with the conclusion, in vertebrate research, that these states are not a uniquely human phenotype. It has been proposed that, because of what is known today about the phylogenetic continuity of the vertebrate brain, the burden of proof is no longer to demonstrate that the higher mammals possess these states, but rather to demonstrate that these animals do not possess these states (see Gray, 2004; Panksepp, 1998). (This conclusion is arguably already warranted on ethical grounds alone.)

In addition, the approach of $\mathrm{K} \& \mathrm{~B}$ complements Passive Frame Theory (Morsella et al., in press), which (a) focuses on the most basic instantiations of these states (but only in humans) and (b) proposes that the primary function of these states is "low-level," controlling basic, 
"integrated" actions (e.g., holding one's breath while underwater; Morsella, 2005). These kinds of actions are selected on the basis of integrating various different kinds of information, including perceptual information and information concerning bodily needs (e.g., visceral urges). In Passive Frame Theory, without consciousness, sophisticated behaviors (e.g., manipulating an object) can arise but the behaviors, being un-integrated, will not be influenced by all the kinds of information by which they should be influenced. In Passive Frame Theory, many of the properties of these states (e.g., the first-person vantage point) arise from the demands of adaptive action selection (see also Merker, 2013).

As noted by $K \& B$, the claim that nonhuman species $X$ must possess conscious states because that species has a certain behavioral capacity depends on various assumptions. This is in part because, in the animal kingdom, the same behavioral capacity (e.g., navigating toward a stimulus) does not always stem from the same underlying mechanisms (e.g., vision vs. echolocation). Even within one species (e.g., humans), the same behavioral operation (e.g., the closing of the eyelids) can be carried out by more than one kind of mechanism (e.g., involuntary blinking versus intentional winking). If one accepts $K \& B^{\prime} s$ assumptions, then one can appreciate that K \& B's (relatively) simple nonhuman model might provide unique insights about the neural underpinnings of consciousness. For example, their arthropod model might provide new data that can resolve the controversy regarding whether conscious states stem from cortical or subcortical structures. (Olfactory consciousness presents challenges for "subcortical" accounts of consciousness; Morsella et al., in press)

In light of K \& B's proposal - in which conscious states are a phenotype shared by many species (including arthropods) - it is interesting to consider that many of the abilities that do distinguish humans from other species (e.g., syntax and co-articulation in speech production) are often mediated unconsciously or may even be consciously impenetrable. This suggests that what makes us conscious is distinct from and more widespread in the animal kingdom than what distinguishes us from other species.

\section{The Action Selection Bottleneck}

$K \& B$ propose that what makes us conscious is shared by other species who face similar challenges regarding the process of action selection. $K \& B$ note that one can perform only one action at a time (e.g., only utter one word at a time). Why do such limitations in the effector system introduce a bottleneck at the action selection stage of processing? According to Cushing et al. (in press), it is because for an overt behavior to be adaptive it must be "integrated." In the architecture proposed by Passive Frame Theory, this means that the overt behavior must be influenced by the level of activation of each of the many (unconscious) response systems that are activated by the contents of the conscious field at one moment in time.

This process has been referred to as "collective influence" (Morsella et al., in press), in which unconscious response systems of the skeletal muscle output system "sample" and are influenced by the contents of the entire conscious field. In such a functional architecture, a bottleneck arises. Action can only be selected sequentially because the summation of activation levels across response systems has to happen within a certain time window (see evidence for such a bottleneck in Pashler, 1993). According to K \& B, such a bottleneck arises in both humans and arthropods; in both species, conscious states enable the bottleneck to function adaptively. $\mathrm{K}$ \& B suggest that the neural underpinnings of such a bottleneck might be easier to investigate in 
arthropods than in humans. If so, then their arthropod model might provide for consciousness research what Aplysia californica provided for the study of learning.

\section{References}

Cushing, D., Ghafur, R. D., \& Morsella, E. (in press). The interdependence between conscious and unconscious processes. In Z. Radman (Ed.), Before consciousness: In search of the fundamentals of mind. United Kingdom: Imprint Academic.

Gray, J. A. (2004). Consciousness: Creeping up on the hard problem. New York: Oxford University Press.

Klein, C., \& Barron, A. B. (2016). Insects have the capacity for subjective experience. Animal Sentience 2016.100.

Merker, B. (2013). The efference cascade, consciousness, and its self: Naturalizing the first person pivot of action control. Frontiers in Psychology, 4, 1-20. doi:

10.3389/fpsyg.2013.00501

Morsella, E. (2005). The function of phenomenal states: Supramodular interaction theory. Psychological Review, 112, 1000-1021.

Morsella, E., Godwin, C. A., Jantz, T. J., Krieger, S. C., \& Gazzaley, A. (in press). Homing in on consciousness in the nervous system: An action-based synthesis. Behavioral and Brain Sciences.

Panksepp, J. (1998). Affective neuroscience: The foundations of human and animal emotions. Oxford University Press.

Pashler, H. (1993). Doing two things at the same time. American Scientist, 81, 48-55. 